Author Affiliation: Boston University School of Medicine, Boston, Massachusetts.

Corresponding Author: David Felson, MD, MPH, Clinical Epidemiology Research and Training Unit, Boston University School of Medicine, 650 Albany St, Ste 200, Boston, MA 02118 (dfelson@bu.edu).

Conflict of Interest Disclosures: None reported.

1. Kyriacou DN, Lewis RJ. Confounding by indication in clinical research. JAMA. 2016;316(17):1818-1819. doi:10.1001/jama.2016.16435

2. Zeng C, Dubreuil M, LaRochelle MR, et al. Association of tramadol with all-cause mortality among patients with osteoarthritis. JAMA. 2019;321(10): 969-982. doi:10.1001/jama.2019.1347

In Reply Dr Felson raises 3 points with regard to a potential role of confounding by indication to explain our findings. ${ }^{1}$

First, he comments on the lack of information on severity of comorbidities. While our propensity score-matched analysis was not able to account for severity level of all covariates, some were incorporated (eg, stages of chronic kidney disease). Nevertheless, a comprehensive propensity scorematched study design with many covariates included tends to balance even unmeasured and unadjusted variables (through their associations with adjusted variables). As such, we expect the unmeasured and unadjustable covariates, such as levels of disease severity, also to be balanced after a comprehensive propensity score matching. Prompted by this comment, we conducted propensity score matching without incorporating stages of chronic kidney disease and found the stages were well balanced among all comparison groups, although they were not included in the propensity score creation. This supports the expectation that other comorbidity severities should be balanced despite not being included in the propensity score generation.

Second, Felson was concerned about the short duration of tramadol use and the intention-to-treat analysis. As we reported, "the mean (range) treatment duration of a prescription for tramadol was 22 (5-67) days...." As such, the mean duration of tramadol use was 110 days, not 22 days. Furthermore, in addition to the intention-to-treat analysis, we conducted an as-treated analysis by censoring follow-up at the time of discontinuation of a patient's medication and found the effect estimates to be stronger than in the intention-to-treat analysis (Table 3 in the article). ${ }^{1}$

Third, Felson raised the issue of the effectiveness of excluding patients with cancer at baseline. As acknowledged in the article, "it is possible that some participants were experiencing pain from undetected early-stage cancer and therefore were given stronger pain medication to relieve the symptoms prior to cancer diagnosis (ie, protopathic bias)." However, we additionally excluded cases of cancer (including all cancer deaths) that occurred within 6 months or 1 year of the start of follow-up and found no material difference in our results (Table 3 in the article), as was done in previous studies. ${ }^{2,3}$

All in all, we adopted a state-of-the-art approach to minimize the potential biases with multiple sensitivity analyses, and our interpretation was balanced. The finding of higher mortality among patients receiving tramadol than patients receiving NSAIDs raises a potential safety concern for tramadol use in patients with osteoarthritis. As we concluded, "however, these findings may be susceptible to confounding by indication, and further research is needed to determine if the association is causal."1

\author{
Guanghua Lei, MD, PhD \\ Hyon K. Choi, MD, DrPH \\ Yuqing Zhang, DSc
}

Author Affiliations: Department of Orthopaedics, Xiangya Hospital, Changsha, Hunan, China (Lei); Division of Rheumatology, Allergy, and Immunology, Massachusetts General Hospital, Boston (Choi, Zhang).

Corresponding Author: Yuqing Zhang, DSc, Division of Rheumatology, Allergy, and Immunology, Department of Medicine, Massachusetts General Hospital, Harvard Medical School, 55 Fruit St, Boston, MA 02114 (yzhang108@mgh.harvard.edu).

Conflict of Interest Disclosures: None reported.

1. Zeng C, Dubreuil M, LaRochelle MR, et al. Association of tramadol with all-cause mortality among patients with osteoarthritis. JAMA. 2019;321(10): 969-982. doi:10.1001/jama.2019.1347

2. Abrahami D, Douros A, Yin H, et al. Dipeptidyl peptidase-4 inhibitors and incidence of inflammatory bowel disease among patients with type 2 diabetes: population based cohort study. BMJ. 2018;360:k872. doi:10. 1136/bmj.k872

3. Hicks BM, Filion KB, Yin H, Sakr L, Udell JA, Azoulay L. Angiotensin converting enzyme inhibitors and risk of lung cancer: population based cohort study. BMJ. 2018;363:k4209. doi:10.1136/bmj.k4209

\section{Dietary Cholesterol or Egg Consumption and Cardiovascular Outcomes}

To the Editor Based on an analysis of 6 prospective cohorts, Dr Zhong and colleagues ${ }^{1}$ concluded that "higher consumption of dietary cholesterol or eggs was significantly associated with higher risk of incident CVD [cardiovascular disease] and all-cause mortality in a dose-response manner" and noted these findings should be considered "in the development of dietary guidelines and updates." At issue is whether their findings, which differ from some previously published meta-analyses on this topic, ${ }^{2,3}$ are sufficiently robust to be considered in guidelines.

Baseline characteristics of study participants, according to 5 levels of reported dietary cholesterol or egg intake, were presented in eTables 1 and 2 in the online supplement. Individuals with the lowest cholesterol intake reported exceptionally low energy intake (902 kcal/d), despite having a similar body mass index as the others. Less alcohol consumption and current smoking and greater use of antihypertensive, lipidlowering, and hormone replacement medications were also associated with lower cholesterol or egg intake.

Based on these differences, we believe those reporting less cholesterol or egg intake may be more health conscious, potentially resulting in general underreporting of total food intake and a more specific bias of underreporting egg intake (during baseline data collection for 5 of 6 study cohorts, low cholesterol or egg intake was recommended ${ }^{4}$ ). Individuals with greater health consciousness may also have been more likely to adhere to prescribed medications and engage in other healthful behaviors that contributed to improved outcomes. ${ }^{5}$

Although the authors adjusted for measured characteristics that may serve as proxies for health consciousness, such 
as baseline alcohol and tobacco use, health consciousness is a latent concept, and it is not clear that the variables adjusted for were sufficient to control for all confounding. In these situations, it is often helpful to use falsification tests, which seek to determine if an association that should not be present after sufficient adjustment is in fact present. In this case, examining whether individuals reporting lower dietary cholesterol or egg intake had higher rates of recommended cancer screenings or immunizations, adjusting for the same factors as in the primary outcome models, could serve as a good falsification test. Dietary cholesterol should not have an effect on completing colorectal cancer screening, for example, so if an association were observed, it would suggest residual confounding by a factor, such as health consciousness, that could serve as a common cause of both lower reported cholesterol or egg consumption and fewer cardiovascular events.

Thomas Charles Keyserling, MD, MPH

Seth A. Berkowitz, MD

Carmen D. Samuel-Hodge, $\mathrm{PhD}$

Author Affiliations: University of North Carolina at Chapel Hill.

Corresponding Author: Thomas Charles Keyserling, MD, MPH, Division of General Medicine and Clinical Epidemiology, Department of Medicine, University of North Carolina at Chapel Hill, 1700 Martin Luther King Jr Blvd, CB 7110, Chapel Hill, NC 27599 (jato@med.unc.edu).

Conflict of Interest Disclosures: None reported.

1. Zhong VW, Van Horn L, Cornelis MC, et al. Associations of dietary cholesterol or egg consumption with incident cardiovascular disease and mortality. JAMA. 2019;321(11):1081-1095. doi:10.1001/jama.2019.1572

2. Berger S, Raman G, Vishwanathan R, Jacques PF, Johnson EJ. Dietary cholesterol and cardiovascular disease: a systematic review and meta-analysis. Am J Clin Nutr. 2015;102(2):276-294. doi:10.3945/ajcn.114.100305

3. Alexander DD, Miller PE, Vargas AJ, Weed DL, Cohen SS. Meta-analysis of egg consumption and risk of coronary heart disease and stroke. J Am Coll Nutr. 2016;35(8):704-716. doi:10.1080/07315724.2016.1152928

4. Krauss RM, Deckelbaum RJ, Ernst N, et al. Dietary guidelines for healthy American adults: a statement for health professionals from the Nutrition Committee, American Heart Association. Circulation. 1996;94(7):1795-1800. doi:10.1161/01.CIR.94.7.1795

5. Rodriguez F, Maron DJ, Knowles JW, Virani SS, Lin S, Heidenreich PA. Association of statin adherence with mortality in patients with atherosclerotic cardiovascular disease [published online February 13, 2019]. JAMA Cardiol. doi 10.1001/jamacardio.2018.4936

In Reply Dr Keyserling and colleagues commented that the observed associations of dietary cholesterol or egg consumption with incident cardiovascular disease and all-cause mortality in our study might be partly attributable to residual confounding due to unmeasured health consciousness. ${ }^{1}$ We agree that by adjusting for several available proxies for health consciousness, we may not have fully eliminated the related confounding.

Their argument originated from a result presented in eTable 1 in the online supplement. A small subgroup ( $\mathrm{n}=1989$ [6.7\%]) who consumed the lowest amount of dietary cholesterol $(<100 \mathrm{mg} / \mathrm{d})$ reported very low energy intake with a median of approximately $900 \mathrm{kcal} / \mathrm{d}$. There are several reasons for this. First, the proportion of women in this small subgroup (66.4\%) was the highest among all the 5 subgroups. It is known that women, on average, consume less total energy than men. In our data set, the median energy intake was 1545 (interquartile range, 1165-2027) kcal/d in women and 1860 (interquartile range, 1399-2502) kcal/d in men. Second, the mean age of this small subgroup (57.7 years) was the oldest among all the 5 subgroups. Older adults ( $\geq 60$ years) generally have considerably lower energy intake compared with younger adults (20-59 years). ${ }^{2}$ Third, dietary cholesterol is found in all foods of animal source such as meat, poultry, eggs, dairy products, fish, and shellfish. People who consume only a small amount of these foods may be likely to have a relatively lower energy intake compared with those who consume more. Fourth, as pointed out by Keyserling and colleagues, underreporting is likely for self-reported dietary intake. However, we do not know who underreported for what reasons.

Our pooling project was originally created to study longterm risks and development patterns of cardiovascular disease over the life course in adults. Our pooled data set did not include variables that are likely unrelated to dietary cholesterol or egg consumption, such as cancer screenings, immunizations, trauma, and respiratory infection. Therefore, we are unable to perform falsification testing. Nonetheless, in a sensitivity analysis, we restricted the entire study sample ( $N=29615$ ) to nonsmokers with body mass index less than 25 and little alcohol intake $(<0.5 \mathrm{~g} / \mathrm{d})$, who may be more health conscious than others. A nonsignificant association with incident cardiovascular disease in the same direction as the main analysis was observed for dietary cholesterol consumption (adjusted hazard ratio per $300 \mathrm{mg} / \mathrm{d}, 1.40$ [95\% CI, 0.96-2.05]) and for egg consumption (adjusted hazard ratio per half an egg per day, 1.20 [95\% CI, 0.99-1.46]). The statistical nonsignificance was probably due to the low power ( $\mathrm{n}=2549$ [8.6\%]).

Future nutritional epidemiologic studies that deliberately include data collection intended for falsification testing are encouraged. It should be proposed a priori in the study design stage to avoid post hoc data mining. However, the absence of an association between a primary exposure and an unrelated outcome might offer indirect support for but certainly not guarantee the existence of an association between a primary exposure and a primary outcome. ${ }^{3}$

\section{Victor W. Zhong, PhD \\ Linda Van Horn, PhD \\ Norrina B. Allen, PhD}

Author Affiliations: Division of Nutritional Sciences, Cornell University, Ithaca, New York (Zhong); Department of Preventive Medicine, Northwestern University Feinberg School of Medicine, Chicago, Illinois (Van Horn, Allen).

Corresponding Author: Victor W. Zhong, PhD, Division of Nutritional Sciences, Cornell University, 315 Savage Hall, 244 Garden Ave, Ithaca, NY 14853 (wenze.zhong@cornell.edu).

Conflict of Interest Disclosures: None reported

1. Zhong VW, Van Horn L, Cornelis MC, et al. Associations of dietary cholesterol or egg consumption with incident cardiovascular disease and mortality. JAMA 2019;321(11):1081-1095. doi:10.1001/jama.2019.1572

2. Ford ES, Dietz WH. Trends in energy intake among adults in the United States: findings from NHANES. Am J Clin Nutr. 2013;97(4):848-853. doi:10. 3945/ajcn.112.052662

3. Prasad V, Jena AB. Prespecified falsification end points: can they validate true observational associations? JAMA. 2013;309(3):241-242. doi:10.1001/jama. 2012.96867 